\title{
A Personalized Machine-Learning-enabled Method for Effi- cient Research in Ethnopharmacology. The case of Southern Balkans and Coastal zone of Asia Minor
}

\author{
Evangelos Axiotis ${ }^{1 *}$, Andreas Kontogiannis ${ }^{2}$, Eleftherios Kalpoutzakis ${ }^{1}$ and George Giannakopoulos ${ }^{3,4}$ \\ 1 Department of Pharmacognosy and Natural Products Chemistry, Faculty of Pharmacy, National and Ka- \\ podistrian University of Athens, axiotisevan@pharm.uoa.gr ; elkalp@pharm.uoa.gr \\ 2 School of Electrical and Computer Engineering, National Technical University of Athens, Greece; \\ andr.kontog@gmail.com \\ 3 Software and Knowledge Engineering Lab, NCSR “Demokritos”, Athens, Greece, ggianna@iit.demo- \\ kritos.gr \\ 4 SciFY PNPC, Greece \\ * Correspondence: axiotisevan@pharm.uoa.gr ; Tel.:00306944934227
}

\begin{abstract}
An ethnopharmacology expert faces several challenges when identifying and retrieving documents and resources related to their scientific focus. The volume of sources that need to be monitored, the variety of formats utilized, the different quality of language use across sources, present some of what we call "big data" challenges in the analysis of this data. This study aims to understand if and how the expert can be supported effectively through intelligent tools for the ethnopharmacological research in the Southern Balkans and Coastal zone of Asia Minor. Our work follows an "Expert-Apprentice" paradigm in a crawling process, where the apprentice is a Machine Learning (ML) algorithm, utilizing a combination of Active Learning (AL) and Reinforcement Learning (RL), and the Expert is the human researcher. ML-powered research improved 3.1 times the effectiveness and 5.14 times the efficiency of the domain expert, fetching a total number of 420 relevant ethnopharmacological documents in only 7 hours versus an estimated 36-hour human-expert effort. Therefore, utilizing Artificial Intelligence (AI) tools to support the researcher can boost the efficiency and effectiveness of the identification and retrieval of appropriate documents.
\end{abstract}

Keywords: Ethnopharmacology; Artificial Intelligence; Web Crawling; Active Learning; Reinforcement Learning; Text Mining; Big Data

\section{Introduction}

Ethnopharmacology is an interdisciplinary field of research based both on anthropological and scientific approaches [1]. The development of a standard scientific approach to retrieve information from the empirical use and define a pharmacological value from traditional preparations must be considered a highly complex and challenging task, strongly filtered by the evolution of human history [2].

In the Southern East European region, ethnobotanical studies are of great interest due to political and economic shifts that have influenced local lifeways, economies, foodways, and transmission of traditional knowledge regarding local health-related practices. [3].

The challenge of discovering and enriching a body of knowledge with pre-existing scientific research has been a persistent need of the scientific community. Nowadays, intelligent systems known as focused crawlers [4], have supported domain experts in 
personalized search. Such approaches combine the power of the search engines with the user's explicit feedback to identify the documents that maximally relate to the interest of the expert. The crawler leverages a limited set of keywords, provided by the users, to retrieve relevant documents. The experts, then, select the ones related to their interest and feed these back to the crawler. With subsequent iterations, the crawler can identify new keywords and fetch more pertinent documents by improving its searches.

Recent works employed data mining techniques to identify ethnopharmacology-related knowledge [5]. However, no work has yet provided personalized, adaptive, realtime support to experts. The present study focuses on the classification of the ethnopharmacological knowledge of Greece, southern Balkans, and the coastal zone of Asia Minor (Figure.1), with the broader aim to introduce a personalized computational approach to biomedical mining as an effective scientific tool for research in ethnopharmacology.

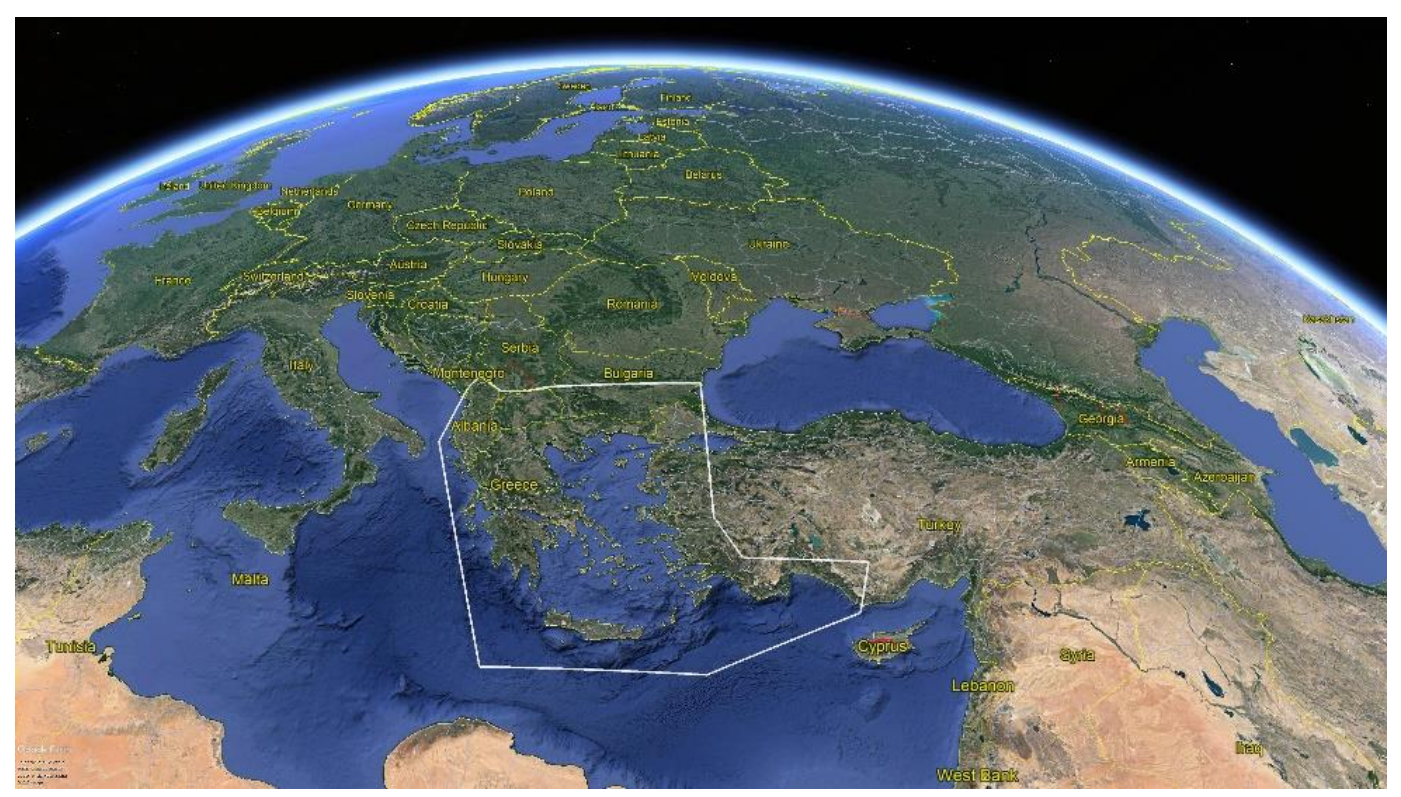

Figure 1. The zone of ethnopharmacological interest in white. Southern Balkans and coastal zone of Asia Minor.

This approach applies ML techniques, to get (a) automated inference on the explicit and implicit interests of the expert, (b) optimization of the crawling process to minimize the feedback of the expert on the appropriateness of retrieved documents.

\section{Materials and Methods}

\subsection{Method Overview}

Our work follows an "Expert-Apprentice" paradigm. The Expert holds explicit and implicit interests. The Apprentice supports the Expert, by learning their interests in two ways. First, the Expert explicitly provides examples of documents, called seeds. Second, over time the Apprentice periodically requests feedback from the Expert for an - ideally minimal - number of candidate documents. The Expert then labels them as interesting or not. The Apprentice resumes their work iteratively until they retrieve a specific number of documents.

In our AI setting, as shown in the Flow diagram (Figure 2), the Apprentice is an ML algorithm that undertakes 2 tasks. In the first task, the algorithm understands the interests of the user (Expert) through explicit feedback (labels of documents as interesting or not). Here, we utilize an ML model deploying pool-based AL for a binary classification 
task, with the Expert being the Oracle (human annotator) during the learning process. In the supervised pool-based AL setting, a model is trained on an initial small labeled training set of relevant and some irrelevant documents. Then, it queries the Oracle with the documents that are predicted to be the most informative for the model from a bigger unlabeled dataset, which is called the pool. After the Oracle has given the corresponding labels for these samples, the training set is augmented with them and the model retrained utilizing the updated data. This training process resumes iteratively until a predefined number of queries ("budget") has been addressed to the Oracle. We note that AL has already been used in other biomedical text mining applications [6,7], where classic ML classification algorithms, such as SVM and Logistic Regression, were examined. In our work, we utilize a neural network (LSTM) as the classification model for the AL setting.

In the second task, the Apprentice is an RL agent that discovers a strategy - policy of crawling documents. The aim of the agent is to minimize the number of retrieved documents, while maximizing the number of relevant ones. To this end, the agent tries to connect the documents fetched so far with the decision of which candidate document to fetch next. We consider that we gather candidate documents from the references of each fetched publication. Every few fetched publications, the algorithm examines how well the strategy did in bringing relevant documents by using the trained AL model. The algorithm then updates its strategy, based on this feedback, trying to improve its decisions in future crawling steps.

\subsection{Defining the Relevant Topics}

The relevant topics of our publication search are defined by the Expert. In our case, the relevant topics referred to ethnopharmacology in Balkan countries and Asia Minor with emphasis on certain plant families and species. More specifically, our domain experts pointed out 31 of the most important plant families. Using the taxonomy of angiosperms published on Flora of Greece [8], we managed to extract all species names from these families. Thus, we constructed a taxonomy of 578 keywords based on geographical locations and plant families.

\subsection{Dataset}

In the selected ethnopharmacology setting, we first examined whether two different researchers agree on the definition of relevance. This would imply that the topic of interest has been sufficiently described to gain common understanding between experts. To this end, we requested them to provide a list of 25 relevant documents - seeds - identified by their URLs. Based on these seeds, we identified a total of 427 documents, which were extracted from the references of them.

We also retrieved another 800 publications, with no prior knowledge of whether they would be related to the topic at hand. This was achieved by a crawling run, which randomly followed references appearing in visited publications, through uniform sampling. By removing duplicates, we ended up with a total of 1012 documents, in addition to the seeds.

We arbitrarily selected a total of 50 documents, of which almost $50 \%$ were part of the seed set (very relevant). Then we asked independently the 2 domain experts to label the documents on a scale from 1 to 4 ( 1 = "highly related" and $4=$ "irrelevant"). We then measured the degree of the inter-annotator agreement through three methods: Raw Agreement (RA), Cohen's kappa (CK), and Krippendorff's alpha (KA), which all showed substantial or good agreement between judges (RA: 0.82, CK: 0.71, KA: 0.92). This clearly showed that the experts do hold a common understanding of what is related to the 
domain of focus. Thus, the senior of the two experts undertook that annotation of data in the next experiments. The rate of annotation across experts was about 5 documents per minute, described only by their titles and abstracts. Thus, the annotation of the total 1012 documents by a single expert would have taken about 200 minutes. We note that this collection of documents would be the pool for our pool-based AL setting.

We now possess a means to obtain "gold-standard" opinions on the relevance of a given document to our domain of interest. We can, thus, employ AL and crawling and evaluate how well the system (a) infers the interests of the expert(s), (b) optimize the crawling process to minimize the number of documents it needs to retrieve.

\subsection{Using Active Learning to Infer Expert Interest}

For the first aim, i.e., inferring what the expert considers related to the topic of interest, we trained an LSTM model with AL, which implements part of the "Expert-Apprentice" workflow we described. Essentially, in our case, it refers to the algorithm which classifies a given document as relevant or not to the interest of the Expert. For this process, we set the budget of queries equal to 250 , i.e., we can only ask the expert their opinion on a maximum of 250 documents. The document pool consists of the 1012 unlabeled documents collected using the random crawling run and those extracted from the seeds. Our LSTM uses a Mean Pooling layer to average the hidden state vectors of all timesteps, i.e., words in a document. This layer is connected to two fully connected layers. The AL model selects from a pool those $\mathrm{k}$ documents for which the corresponding classification probabilities are the $\mathrm{k}$ smallest. In order our model to output probability values for each corresponding class, we use the Softmax as the activation function of the output layer. We arbitrarily use $\mathrm{k}=10$. As input, we use the pre-trained word2vec word embeddings of each document, based on the bio.nlplab.org embedding [9].

Next, we tried to understand if the system would help the expert to retrieve a sufficient number of related documents under a significantly reduced human time allocation. To this end, we run 4-fold-cross-validation (4 experiments). In each AL experiment, the training set was initially composed of 23 relevant and 27 irrelevant documents, for a total of 50 documents. In each run, we kept 100 held-out documents, evaluating the performance of the AL prediction: 50 were related and 50 were not related to the topic at hand. We essentially asked the expert about 250 documents (vs. 1012 that he would have needed to evaluate if no active learning was employed), reducing the required time and effort by approximately $75 \%$. For this level of reduction, the AL managed to classify correctly 88 out of 100 documents on average (88\% accuracy). 


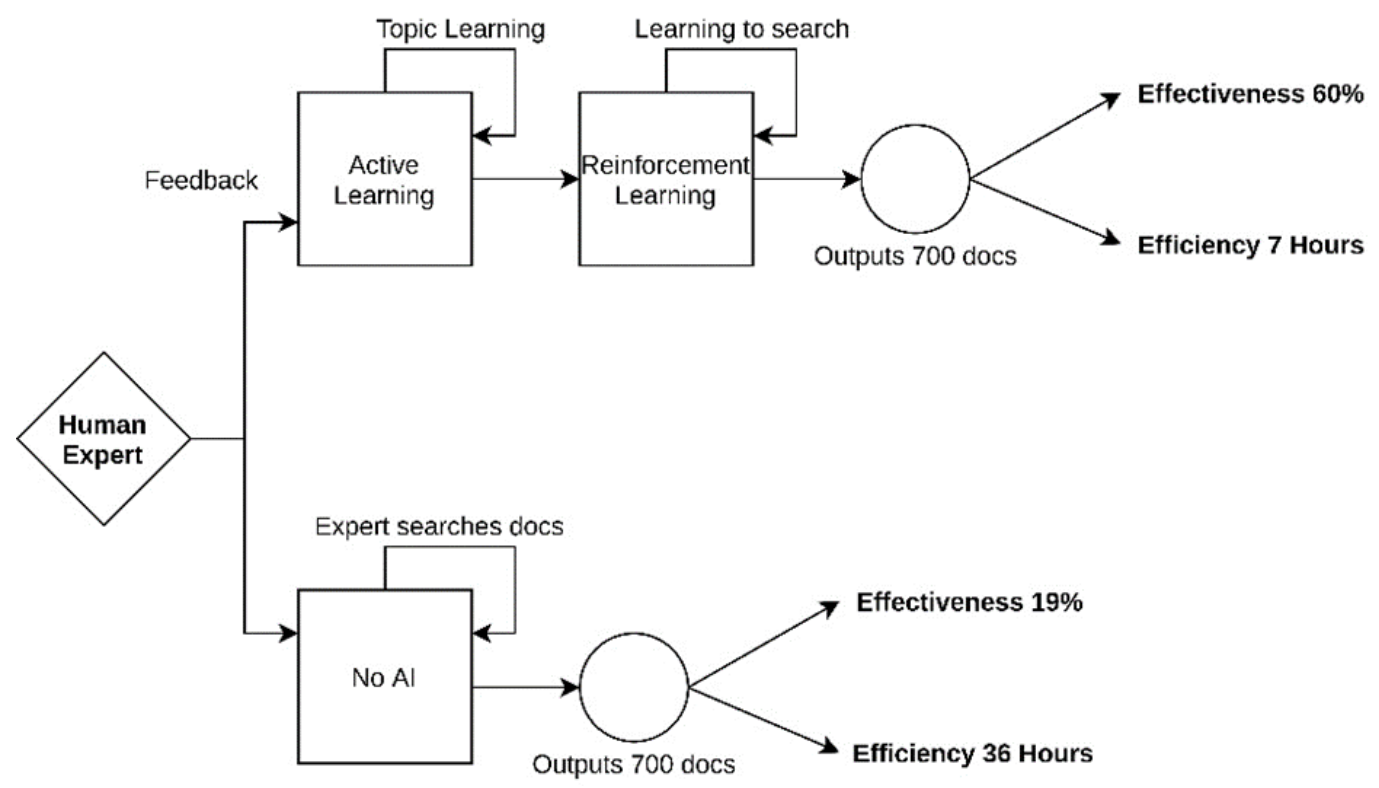

Figure 2. Flow diagram of the experimental method

\subsection{Reinforcement Learning}

In our setting, an RL algorithm allows the crawler to determine a policy, so that it retrieves a fixed number of documents while maximizing the number of related ones. Recently, there have been approaches of focused crawling [10] and biomedical data mining [11] with RL. We consider that the agent - the crawler - fetches a new URL at each timestep and exists in a crawling environment, which provides states, actions, and rewards. Each time ( $t$ ) the agent fetches a new URL, as a result of an action selection (At), then it transitions from the current state $(\mathrm{St})$ to another state $(\mathrm{St}+1)$ and observes a reward (Rt). The states are related to the history of information (number of relevant and irrelevant URLs) fetched by the crawler. The actions are related to the URLs (keywords found on the anchor text) extracted from a state transition. The reward is related to the relevance of the current fetched publication with the defined topic. We set the reward equal to 1 for relevant publications and 0 otherwise. For the reward function, at first, we use the LSTM trained by $\mathrm{AL}$ in order to decide whether a document is related to ethnopharmacology. Then, we deterministically filter the related predicted ones using the taxonomy of keywords constructed.

The goal of the agent is to find a policy, to maximize the discounted cumulative received reward Gt $=\mathrm{Rt}+\gamma \mathrm{Rt}+1+\gamma 2 \mathrm{Rt}+2+\ldots+\gamma \mathrm{T}-\mathrm{tRT}$, where $\mathrm{T}$ is the fixed number of total documents that the crawler should fetch and $\gamma$ is the discount factor. For our experiment, we arbitrarily set $\mathrm{T}=700$ and $\gamma=0.99$.

Our evaluation measure for focused crawling is the harvest rate $\mathrm{HR}(\mathrm{t})$ [4], which is the cumulative percentage of relevant fetched documents up to timestep $t$.

We employ a Deep Q-learning approach, utilizing the Deep Q-Network (DQN) agent [12], which is based on the TD Error, $\mathrm{Rt}+1+\operatorname{maxaQ} \pi^{\prime}(\mathrm{St}+1, \mathrm{a} ; \theta-)-\mathrm{Q} \pi(\mathrm{St}, \mathrm{At} ; \theta)$, where $\mathrm{Q} \pi$ and $\mathrm{Q} \pi^{\prime}$ are the action-value functions under the policies $\pi$ and $\pi^{\prime}$, respectively. That is $\mathrm{Q} \pi(\mathrm{St}, \mathrm{At})=\mathrm{EU}(\mathrm{D})\left[\mathrm{Rt}+1+\operatorname{maxaQ} \pi^{\prime}(\mathrm{St}+1, \mathrm{a} ; \theta-) \mid \mathrm{St}, \mathrm{At}\right]$. The DQN agent consists of two neural networks with the same architecture - a Q-Network $(\theta)$ and a Target Q-Network ( $\theta-)$ - in order to approximate $\mathrm{Q} \pi$ and $\mathrm{Q} \pi^{\prime}$, respectively. Additionally, it has a replay buffer D, called Experience Replay, which is important for uniform sampling mini-batches of uncorrelated past state transitions. For each Q-Network, we utilize a Multilayer perceptron (MLP) with two hidden layers. We initialize the Experience Replay with a priori 
experience given from seeds, all of which are highly relevant documents, in order to speed up the training process. Using Deep Q-learning, we essentially face a regression problem, minimizing the Mean Square Error of TD Error with respect to $\theta$.

Moreover, to balance the Exploration-Exploitation dilemma, calling us to decide between always choosing the best action (exploiting) and uniformly selecting sometimes one (exploring), we use an $\varepsilon$-greedy policy for sampling, i.e., action selection. That is, the best action of a given state is chosen with probability $1-\varepsilon$, otherwise a random one is selected (with probability $\varepsilon$ ). As training progresses, $\varepsilon$ diminishes over time by a factor of $\lambda$ until it reaches a defined value $\varepsilon F$. Formally, $\varepsilon=\max \{\varepsilon F, \lambda \varepsilon\}$. We set $\lambda=0.99$, initial $\varepsilon 0=0.15$ and $\varepsilon \mathrm{F}=0.03$.

Finally, for our agent to be able to select URLs - related to actions - extracted from past state transitions, we use a priority queue, called the frontier, so that the best action is selected in $\mathrm{O}(\log \mathrm{N})$; where $\mathrm{N}$ is the frontier size. We note that a URL is stored into the frontier along with its corresponding Q-value, which has been estimated by the Q-Network. Also, we define another structure, called Closure, where we store fetched URLs, so that the agent will not fetch them again.

\section{Results}

\subsection{Ethnopharmacological Inference}

Ethnobotany in the Southern East (SE) European region includes local traditional knowledge from countries such as Albania [13], FYROM [14], Bulgaria [15], and Greece $[16,17,18]$. In the present study, the coastal zone of Asia Minor is included $[19,20,21]$. The conspicuous floristic affinities of the East Aegean islands with neighboring western Anatolia, along with the enduring influence that Anatolian Turks had on eastern Europe during the Ottoman empire, prompted us to compare data of ethnopharmacological studies from this area.

The Balkan area can be described both as a "linking bridge" of cultures and as a violent transitional zone between civilizations; the bio-cultural-historical amalgam of races in the southern part of the peninsula represents the core of "Balkanization" [22], a concept coined to define the anthropological mixture in SE.

Moving towards the southern parts of the peninsula, a unique cultural and linguistic pattern has evolved with populations influenced by the dominance of ancient Macedonians (500-168 BC), Romans (168-284 BC), Byzantines (395-1453 AD), and Ottomans (1299$1922 \mathrm{AD}$ ). From the beginning of the 19th century, the Balkans were transformed from protectorates of foreign empires to independent countries, but the cultural amalgam was so intertwined that was embodied by the borders of these nation-states even after many generations. Even if hundreds of different ethnic groups exist in these countries, they are incorporated into the local societies in such a way that it is very difficult to investigate their origin [23]. In many instances, researchers described an erosion of traditional medical knowledge due to deep social changes [3]. As a result, the loss of information is inevitable.

Moreover, rich biodiversity characterizes these regions and a great number of species have been used in traditional medicine. A non-exhaustive list of species in the earliest written records still preserved has been exploited by local healthcare [24].

Lately, many online resources are trying to pass on this knowledge, mostly accounting for oral reports from elderly people. These attempts create a conspicuous variety of sources that needs new technologies to be processed [25], classified and validated, for the 
best advantage of the scientific community. In our project, we were faced with this great challenge. The volume of sources that needed to be monitored exceeded a database of 10,000 identified references, based on the topics summarized in Table 1 . We limited the Plant Families in the classification of Angiosperms and from these we considered 31 of the most important plant families used in ethnopharmacology. Furthermore, the part of the plant used, uses and recipes, MeSH terms, and geographical regions, were used to filter the identified references.

Table 1. Ethnopharmacological Topics / Mesh Terms used for our setting

\begin{tabular}{|c|c|c|c|c|}
\hline Plant Families & Part of Plant used & Uses / Recipes & MeSH Terms & Geographical Regions \\
\hline Alliaceae & Aerial Part & Decoction & Greek ethnopharmacology & Albania \\
\hline Anacardiaceae & Flower & Infusion & Traditional greek medicine & FYROM or Northern Macedonia \\
\hline Apiaceae & Chalices of flowers & Maceration & Natural product & Bulgaria (southern) \\
\hline Asparagaceae & Seed & Powder & Medicinal plant & Greece \\
\hline Asphodelaceae & Leaf & Juice & Plant extracts & coastal zone of Turkey or Asia Minor \\
\hline Asteraceae & Fruit & Poultice & Pharmacological action & \\
\hline Boraginaceae & Stem & tsp of oil & Disease & \\
\hline Brassicaceae & Bark & Paste & Treatment & \\
\hline Cactaceae & Root & Whole plant preparation & Antimicrobial activity & \\
\hline Cannabaceae & Clove & Cook & Radical scavenging activity & \\
\hline Capparaceae & Stigma & Raw & Antioxidant activity & \\
\hline Cistaceae & Bulb & Milk & Ethnobotany & \\
\hline Fabaceae & Foliage & Solvent / adjuvant used & Pharmacognosy & \\
\hline Fagaceae & Shoot & Honey & Herbal medicine & \\
\hline Gentianaceae & Branch & Wine / Water & Greek folk medicine & \\
\hline Hypericaceae & Whole Plant & Filtrate & Home remedies & \\
\hline Lamiaceae & Wooden & Pounded & Folk remedies & \\
\hline Liliaceae & Kernel & Extract & Materia medica & \\
\hline Malvaceae & Fiber & Dried & Phytotherapy & \\
\hline Moraceae & Rhizome & Fresh & Southern Balkans & \\
\hline Myrtaceae & Ground plant & Soup & Balkans & \\
\hline Oleaceae & Petioles & Soaked in & Albanian ethnopharmacology & \\
\hline Paeoniaceae & Stem bark & Milled & Bulgarian ethnopharmacology & \\
\hline Platanaceae & Tuberous root & Mixed with & Southern Bulgary ethnopharmacology & \\
\hline Rosaceae & Styles & Warm and smoke & FYROM ethnopharmacology & \\
\hline Salicaceae & Latex & Chew & Northern Macedonia ethnopharmacology & \\
\hline Scrophulariaceae & Gum & Swallow & Turkish ethnopharmacology & \\
\hline Solanaceae & Peels & Bake & Turkish coastal zone ethnopharmacology & \\
\hline Urticaceae & Ripe ears & Bandage & Turkish folk medicine & \\
\hline Valerianaceae & Hard wood & Squeez & Southern Balkans folk medicine & \\
\hline Vitaceae & Radix & Disperse in & Pharmacotherapy & \\
\hline
\end{tabular}

\subsection{Crawling Results}

In a baseline setting, automatic crawling would just exhaustively return the references of the seeds, and then recursively the references of these references. This causes a significant growth in the number of fetched documents, without ascertaining quality results. A human, on the other hand, would follow a much more targeted approach, by evaluating the most promising documents each time, visiting them, and in turn, judging their references. In the RL setting, the agent may determine that in some cases it is promising to follow a marginally relevant reference, to then reach a wealth of other publications that might have not been fetched with the previous method.

In this case, we measure the reduction in crawled publications, compared to the baseline. We also take into account how many documents retrieved were indeed relevant to our topic. We note that in the baseline approach:

- in the first 25 documents, we have approximately 850 references to visit;

- in the first 700 fetched documents, the identified references are approximately 10,000.

We have estimated, by sampling 50 representative documents, that the percentage of related references per document is approximately $19 \%$. On the other hand, our DQN agent fetched 700 documents, measuring the $\mathrm{HR}$ as 60\% (420 relevant documents from 700), i.e., improving 3.1 times the effectiveness over the baseline. 
As a second aspect, we examined the same number (420) of related documents the expert can retrieve in the unit of time. Taking into account the time needed for the expert to annotate a single document, we estimate that they need a total time of 36 hours for this task, which is a rate of 13 relevant documents per hour. The RL-based system achieved a rate of 68 relevant documents per hour through a 7-hour crawling task, and thus improved 5.14 times the efficiency over the expert.

\title{
5. Conclusions
}

In this study, we demonstrated that the use of ML, utilizing AL and RL methods, can significantly boost the effectiveness and efficiency of ethnopharmacology researchers. Moreover, we demonstrated that AI-powered research can improve 3.1 times the effectiveness and 5.14 times the efficiency of the domain expert, suggesting the use of such tools for ethnopharmacology research. After this preliminary study, we can hypothesize that the use of Artificial Intelligence tools can indeed support the researchers to boost the efficiency and effectiveness of the identification and retrieval of appropriate documents.

\begin{abstract}
Author Contributions: Conceptualization, E.A., A.K. and G.G.; methodology, E.A., A.K., G.G; software, A.K. and G.G.; validation, E.A., A.K. and G.G.; formal analysis, X.X.; investigation, X.X.; resources, X.X.; data curation, X.X.; writing-original draft preparation, X.X.; writing-review and editing, X.X.; visualization, X.X.; supervision, X.X.; project administration, X.X.; funding acquisition, Y.Y. All authors have read and agreed to the published version of the manuscript.
\end{abstract}

Funding: Please add: “This research received no external funding

Conflicts of Interest: The authors declare no conflict of interest.

\section{References}

1. Heinrich, M.; Jäger, A. K. Ethnopharmacology, John Wiley \& Sons: Chichester, UK, England, 2015

2. 2. Lukman, S.; He, Y.; Hui, S.C. Computational methods for Traditional Chinese Medicine: A survey. Comput. Methods Programs Biomed. 2007, 88, pp. 283-294. https://doi.org/10.1016/j.cmpb.2007.09.008

3. 3. Quave, C.L.; Pardo-De-Santayana, M.; Pieroni, A. Medical ethnobotany in Europe: From field ethnography to a more culturally sensitive evidence-based cam? Evid-Based Compl. Alt. 2012. https://doi.org/10.1155/2012/156846

4. 4. Chakrabarti, S.; Van den Berg, M.; Dom, B. Focused crawling: a new approach to topic-specific Web resource discovery. Computer networks 1999, 31, pp. 1623-1640.

5. 5. Yadong, Z.; Kongfa, H.; Tao, Y. Mining effect of Famous Chinese Medicine Doctors on Lung-cancer based on Association rules. IEEE International Conference on Bioinformatics and Biomedicine (BIBM) 2019, pp. 2036-2040.

6. 6. Naseem, U.; Khushi, M.; Khan, S.K.; Shaukat, K.; Moni, M.A. A Comparative Analysis of Active Learning for Biomedical Text Mining. Appl. Syst. Innov. 2021, 4, 23. https://doi.org/10.3390/asi4010023

7. 7. Chen, Y.; Mani, S.; Xu, H. Applying active learning to assertion classification of concepts in clinical text. J. Biomed. Inform. 2012, 45, pp. 265-272. https://doi.org/10.1016/j.jbi.2011.11.003

8. 8. Flora of Greece. Vascular Plant Checklist of Greece. Available online: http://portal.cybertaxonomy.org/flora-greece/ (accessed on 31 March 2021)

9. 9. Biomedical natural language processing. Tools and resources. Available online: https://bio.nlplab.org/ (accessed on 31 March 2021)

10. 10. Han M.; Wuillemin PH.; Senellart P. Focused Crawling Through Reinforcement Learning. In Web Engineering. ICWE. Lecture Notes in Computer Science: Mikkonen T., Klamma R., Hernández J., Eds.; Springer, Cham., 2018 , Vol 10845. https://doi.org/10.1007/978-3-319-91662-0_20

11. 11. Souid, A.; Sakli, N.; Sakli, H. Classification and Predictions of Lung Diseases from Chest X-rays Using MobileNet V2. Appl. Sci. 2021, 11, pp. 2751. https://doi.org/10.3390/app11062751

12. 12. Mnih, V.; Kavukcuoglu, K.; Silver, D. et al. Human-level control through deep reinforcement learning. Nature 2015, 518, pp. 529-533 https://doi.org/10.1038/nature14236

13. 13. Pieroni, A. Local plant resources in the ethnobotany of Theth, a village in the Northern Albanian Alps. Genet. Resour. Crop Evol. 2008, 55, pp.1197-1214. https://doi.org/10.1007/s10722-008-9320-3

14. 14. Miskoska-Milevska, E.; Stamatoska, A.; Jordanovska, S. Traditional uses of wild edible plants in the Republic of North Macedonia. Phytol. Balc. 2020, 26, pp.155-162.

15. 15. Ivanova, T.A.; Bosseva, Y.Z.; Ganeva-Raycheva, V.G.; Dimitrova, D. Ethnobotanical knowledge on edible plants used in zelnik pastries from Haskovo province (Southeast Bulgaria). Phytol. Balc. 2018, 24, pp. 389-395. 
16. 16. Vokou, D.; Katradi, K.; Kokkini, S. Ethnobotanical survey of Zagori (Epirus, Greece), a renowned centre of folk medicine in the past. J. Ethnopharmacol., 1993, 39, 187-196. https://doi.org/10.1016/0378-8741(93)90035-4

17. 17. Axiotis, E.; Halabalaki, M.; Skaltsounis, L.A. An ethnobotanical study of medicinal plants in the Greek islands of North Aegean Region. Front. Pharmacol.,2018,9, pp.1-6. https://doi.org/10.3389/fphar.2018.00409

18. 18. Tsioutsiou, E.E.; Giordani, P.; Hanlidou, E.; Biagi, M.; De Feo, V.; Cornara, L. Ethnobotanical Study of Medicinal Plants Used in Central Macedonia, Greece. Evidence-based Complement. Altern. Med. 2019. https://doi.org/10.1155/2019/4513792

19. 19. Ugulu, I.; Baslar, S.; Yorek, N.; Dogan, Y. The investigation and quantitative ethnobotanical evaluation of medicinal plants used around Izmir province, Turkey. J. Med. Plants Res. 2009. 3, pp. 345-367. https://doi.org/10.5897/JMPR.9001216

20. 20. Kargioğlu, M.; Cenkci, S.; Serteser, A.; Konuk, M.; Vural, G., Traditional uses of wild plants in the middle Aegean region of Turkey. Hum. Ecol. 2010. 38, pp.429-450. https://doi.org/10.1007/s10745-010-9318-2

21. 21. Polat, R.; Satil, F. An ethnobotanical survey of medicinal plants in Edremit Gulf (Balikesir - Turkey). J. Ethnopharmacol. 2012. 139, pp. 626-641. https://doi.org/10.1016/j.jep.2011.12.004

22. 22. Ballinger, P. Definition Dilemmas: Southeastern Europe as a «Culture Area»? Balkanologie 1999.2. https://doi.org/10.4000/balkanologie

23. 23. Carter, F.W. An Historical Geography of the Balkans, Academic Press: NY, USA, 1977; pp.580.

24. 24. Legakis, A.; Constantinidis, T.; Petrakis, P. V. Biodiversity in Greece, Global Biodiversity 2018. https://doi.org/10.1201/9780429487750-4

25. 25. Yao, Y.; Wang, Z.; Li, L.; Lu, K.; Liu, R.; Liu, Z.; Yan, J. An Ontology-Based Artificial Intelligence Model for Medicine Side-Effect Prediction: Taking Traditional Chinese Medicine as an Example. Comput. Math. Methods Med. 2019. https://doi.org/10.1155/2019/8617503 